\title{
Learning about pain from others: an observational learning account
}

Liesbet Goubert $^{{ }^{*}}$, PhD, Johan W.S. Vlaeyen ${ }^{2,3}, \mathrm{PhD}$, Geert Crombez ${ }^{1}, \mathrm{PhD}$, Kenneth D. Craig ${ }^{4}, \mathrm{PhD}$

1 Department of Experimental-Clinical and Health Psychology Ghent University

Belgium

2 Department of Psychology

Catholic University of Leuven

Belgium

3 Department of Clinical Psychological Science

Maastricht University

The Netherlands

$4 \quad$ Department of Psychology

University of British Columbia

Canada

* Corresponding author: Liesbet Goubert, Department of Experimental-Clinical and Health Psychology, Ghent University, Henri Dunantlaan 2, B-9000 Ghent, Belgium. Tel: +32 (0)9 26462 62, Fax: +32 (0)9 2646489. Electronic mail may be sent to Liesbet.Goubert@Ugent.be.

Number of text pages: 20

Number of words (references included): 5759

Number of references: 81

Number of figures: 0

Number of tables: 0

Category: Focus Article

Key words: pain, learning, observational learning, empathy, pain beliefs

Running title: learning about pain from others 


\begin{abstract}
Although direct experience and verbal instruction are important sources in the development of painrelated beliefs and behaviors, accumulating evidence indicates that observation of others in pain may be equally as important. Taking a contemporary view on learning as a starting point, we discuss available evidence on observational learning in the context of pain, highlight its importance for both development and management of chronic pain problems, and discuss potential moderators of observational learning effects. We argue that the capacity to understand and appreciate the experience of another person is fundamental to observational learning, including use of this information to establish the association between pain and antecedent or consequent stimuli. A main objective of this paper is to stimulate research on the role of learning about pain from others. Several lines for further research, including clinical applications, are delineated.
\end{abstract}

\title{
Perspective
}

Based upon a contemporary view on learning, this focus article delineates how pain-related beliefs and behaviors may be learnt by observing others. It is discussed how further research on the acquisition of pain-related beliefs/behaviors might further our understanding of pain and disability.

\section{Key words}

pain, learning, observational learning, empathy, pain beliefs 


\section{Introduction}

Negative beliefs and misconceptions about pain are central to many psychosocial perspectives on chronic pain and disability ${ }^{8,50}$ and are often targeted as key mechanisms in cognitive-behavioral treatments. ${ }^{42}$ Surprisingly, almost no research is available examining the origins of these pain-related beliefs. Although these beliefs can be learnt by direct experience with pain, they are also prevalent among pain-free individuals. ${ }^{28,29}$ Here, we explore the position that pain-related beliefs and attitudes are acquired by observing others. We take as a starting point a contemporary view on learning and apply this to observational learning in the context of pain. Using this conceptualization, we examine how and when observational learning contributes to the development of pain-related responses and delineate directions for future research.

\section{Observational learning: definition and procedures}

Modern learning theories conceptualize learning as changes in behavior that occur as a consequence of regularities in an individual's environment. ${ }^{19}$ Behavior, here, refers to any observable response, irrespective of whether it is the product of deliberate control (e.g., pressing a button, making a statement), reflexive/automatic processes (e.g., autonomic arousal, nociceptive reflexes), or neuronal activity (e.g., electric brain activity). ${ }^{19}$ This view is consistent with the seminal work of Bandura, ${ }^{4}$ who defined observational learning as changes in patterns of behavior that are a consequence of observing the behavior of others. Through observation of another's behavior in a particular situation, an individual acquires information about that situation and about the consequences of specific actions in that situation. For example, children who observe their parents displaying fear and avoidance reactions to back-stressing activities, such as lifting heavy objects, may adjust their appreciation of that particular situation ("back-stressing activities are dangerous”) and the behavioral consequences (“avoidance of back-stressing activities reduces pain”). This information may translate into changes in attitudes and behavior immediately, e.g., acquisition of unfavourable attitudes to certain activities, or later when pain is experienced by the individual. 
Two possible learning procedures must be considered in observational learning: classical conditioning and operant conditioning. ${ }^{18}$ These conditioning procedures concern observed changes in behavior attributable to the pairing of initially neutral antecedent events and subsequent potent stimuli (classical conditioning), and the impact of associating specific behaviors with reinforcing or punitive consequences (operant conditioning). Illustrative of classical conditioning is the work of Field and colleagues on the role of observational learning in the development of childhood fears ${ }^{1,2}$ : children viewed pictures of novel animals that were followed by pictures depicting scared faces or happy faces. As expected, children came to report being most afraid of the animals that were associated with pictures of the scared faces. Illustrative of operant conditioning is the work of Bandura examining the social context of aggression. ${ }^{3}$ Children observed a video of an adult who violently and aggressively destroyed a large doll. Some of the children witnessed the adult being adversely sanctioned for this behavior and other children witnessed the adult being rewarded. It was observed that children who saw the adult being punished were less likely to imitate the aggression towards a similar doll, relative to those who saw the adult being rewarded, indicating that children learnt about the consequences of the modeled aggressive behavior.

\section{Observational learning and pain}

Investigations of observational learning procedures as sources of beliefs about pain and its treatment, pain-related fears and behavioral responding are limited. Seminal work demonstrating a powerful impact of models who were intolerant or tolerant of pain was undertaken by Craig and colleagues several decades ago (for overviews, see Craig ${ }^{13}$; Hermann ${ }^{38}$ ). Research participants came to match tolerant and intolerant models in both self-report ${ }^{17}$ and nonverbal behavior ${ }^{68}$, although the latter was not as subject to immediate social influence. The social models also had an impact upon autonomic activity and psychophysical measures of sensory processing of painful events ${ }^{15}$, indicating that observational learning influences both observable expression of pain as well as the subjective experience. 
Few experiments have subsequently been undertaken. In an experimental study, ${ }^{25}$ children observing their mothers' reactions during painful exposure of the hand in cold water subsequently displayed lower pain thresholds when their mother had voluntarily exaggerated her pain. Furthermore, children displayed reduced facial displays of pain when the mother had voluntarily suppressed her reaction. An instructive study was provided by Olsson and colleagues, ${ }^{61}$ who examined observational learning of pain-related fear. Participants viewed a video of a model who was exposed to signals that predicted electric shock or safety signals. The model's reactions were visible through facial and bodily display (= observation phase). Subsequently, participants volunteered in an experiment similar to the one observed (= test phase). Findings showed most pronounced skin conductance responses of observers to the stimuli signaling shock to the models in both the observation and test phase. Furthermore, fMRI analyses showed that the amygdala was involved both during learning (observation phase) and expression (test phase) of learned fear. The authors interpreted these findings as evidence for the similarity in neural processes underlying direct and observational fear learning, even though research examining both peripheral autonomic activity and brain imaging demonstrates only partial similarity with the differences having major theoretical importance ${ }^{14,41,60,72}$. While these and other studies provide substantial evidence of a powerful impact of observing others in pain on neurophysiological activity in observers, the broader mechanisms whereby observational learning plays a crucial role in establishing pain-related responses remain relatively uninvestigated; no systematic research is available examining cognitive and affective mechanisms (e.g., changes in beliefs about pain and attitudes toward pain) underlying the effects on behavior, including the moderators of these effects. In this paper, several lines for future research are outlined.

\section{Modulation of observational learning}

In this paper, we argue that the capacity to understand and appreciate the experience of another person, or, in other words, to "empathize" with others is fundamental to observational learning. We previously defined empathy as a sense of knowing the personal experience of the other person, which is a cognitive appreciation that is accompanied by both affective and behavioral 
responses. ${ }^{26,27}$ Some empathic resonance with the model needs to be present for observational learning to take place, whether the reaction is self-oriented fear or other-oriented sympathetic fear. ${ }^{27,80} \mathrm{~A}$ substantial functional neuroimaging literature examining cortical and subcortical reactions when pain is perceived in others indicates that primarily brain areas indicative of affective responses, rather than sensory responses, are engaged in response to another's pain. ${ }^{51,73}$ These responses are not exclusive to humans, and have been demonstrated in non-human species as well, ${ }^{49,67}$ explaining social learning in mammals. ${ }^{11,33}$ In humans, basic automatic/reflexive empathic reactions observed in infants to their mothers develop with maturation and experience, resulting in the sophisticated empathic system observed in adults, in which automatic empathic processes are modulated by social learning experiences and observers' higher level executive functions. ${ }^{16,26,27}$

Whether covert affective and cognitive responses to another's pain translate into overt action (i.e., observable to others) $)^{26,27}$ is dependent upon observed environmental regularities, observer characteristics and their interaction. Environmental and observer characteristics may impact upon the type of information attended to and/or learnt during observational learning, determining if, how and when the novel information is translated into overt behavior. When the observed event relates to the immediate needs of the observer or the observed person, the information commanding attention is likely to be translated right away into overt behavior (e.g., the observed person's reaction signals a high risk of accidental injury or a parent watching his/her child fall off a swing). On the other hand, when the type of information learnt instructs on (characteristics) of the stimulus associated with the other's behavior, the information likely will be stored in memory and not immediately translated into overt behavior (e.g., others are observed using effective pain medication or a child who sees her mother displaying pain behavior during ironing may learn that ironing is painful).

\section{Modulation by characteristics in the environment}

Diverse immediate events are expected to serve as determinants of observational learning during exposure to others in pain, including sources of information about the person in pain and setting events. Setting events include the general context (e.g., work or health care setting) and specific 
events leading up to, associated with, and following the evidence of a painful reaction (e.g., the presence of wounds, sources of injury). Features of the observed person in pain include behavior preceding and following the painful event, the immediate reaction to painful insult, including facial and bodily reactions to potentially painful situations, and the execution of skills that may or may not terminate or palliate the discomfort. Following from our definition of learning, observational learning effects arise from observing relationships between 2 or more environmental stimuli. For example, the combination of a model's facial expression of pain and another environmental event might inform an observer whether the situation threatens him/herself, urging the observer to avoid or even escape that situation and exercise caution thereafter (e.g., a car driving on the adjoining lane and smashing into the crash barrier) or threatens another person, encouraging the observer to assist a person at risk of pain (e.g., removing a child from a swing when another has fallen and hurt herself). ${ }^{30}$

Furthermore, labeling a change in behavior as a consequence of observational learning requires at least two associations to be learnt. Take as an example a child observing his/her mother evidencing a painful face when lifting a shopping bag. For the child to learn that the bag is associated with pain, first, he/she should learn that the painful facial expression of the mother relates to lifting the bag. In this illustration of vicarious classical conditioning, ${ }^{5}$ the facial expression of pain is a biologically relevant and aversive (unconditioned) stimulus, that, if associated with bag lifting, makes the latter a (conditioned) cue signaling danger and evocative of aversive arousal. However, there is a second association that needs to be learnt in order to call the process 'observational' learning. That is, the child should also have learnt an association between the bag and painful properties of lifting the bag, which are inferred by virtue of the facial expression of the mother. This second association sets the occasion for a change in behavior that makes this form of learning "observational".

Observational learning effects are expected to be modulated by the salience and type of the environmental stimuli. Salience would include variations in expressiveness of the model; more vigorous pain or fear behaviors (e.g., reflexive escape, facial grimaces, cry) would be more likely to inform observers that the model is in need, enhancing the learning impact and increasing the 
probability that the observer immediately translates this knowledge into action patterns. In the context of pediatric pain, studies have indeed shown that more expressed distress by infants was associated with more parental reassurance, ${ }^{79}$ bounce, rock and pat. ${ }^{7}$ In children, parental reassurance, criticism and apologizing have been shown to be associated with higher expressed children's distress. ${ }^{53,71}$ The types of pain behaviors observed in others throughout life would be expected to have a cumulative impact on the repertoire of behaviors available to observers when confronted with comparable painful situations.

Future studies should investigate which cues provided by models are particularly salient or used by observers to acquire information about painful settings. Facial displays of pain are potent forms of social communication, ${ }^{78}$ but perhaps total body pain behaviors or displays of fear instead of pain might be even more attention-grabbing and evocative. ${ }^{76}$ Mumme et al. ${ }^{58}$ demonstrated that children responded more vigorously to their mother's vocal expression as compared to facial expression, possibly because acoustic properties of vocal affective expressions such as loudness and pitch may induce emotions more directly. Given that considerable human communication uses verbal channels, it is also of interest to know the differential impact of the models' verbal and non-verbal (facial or body expression, voice quality) cues. Poole and Craig ${ }^{66}$ found that observers attached greater importance to nonverbal as compared to verbal information when judging the credibility of pain expression.

Human sensitivity to social contexts also indicates that how others in the environment react to a model's pain (behavior) might guide observational learning. As observational learning is facilitated by sharing attention, ${ }^{55}$ studies on gaze following, an early component of shared attention, are informative. Indeed, the ability to orient attention rapidly according to the gaze of others informs observers of another's object of interest. ${ }^{40}$ Research has shown that observer's attention is modulated by the observation of gaze direction and by inferences from this observation. ${ }^{59}$ Furthermore, fearful facial expressions have been shown to enhance gaze-triggered attention orienting in observers. ${ }^{75}$ In the context of pain, little research is available on this. However, others' gaze direction, along with their 
facial expressions, might be expected to provide crucial information about pain-related situations. For example, a father's fearful expressions when gazing at the mother's lifting of a heavy bag may signal to an observing child that lifting a heavy bag is dangerous. A striking illustration describes how infants are attuned to their mother's nonverbal emotional displays. Horton and Pillai Riddell ${ }^{39}$ found that during routine needle injections for immunization purposes infants were more likely to attend to their mother's faces if the mother had expressed greater fear prior to the needle stick. Mothers who expressed greater fear also had infants who expressed greater facial displays of distress to the needle stick.

Features of the relationship between the observer and the model also moderate observational learning effects. Family members and other intimates are more likely to serve as models for painrelated responses than strangers, although the benefits of observational learning are not restricted to observing those close to the individual. It can be expected that observational learning will have a larger effect when there is a greater similarity between the model and the observer. ${ }^{10}$ Observational learning effects are likely to be greater when observers belong to the same "in group" as the model (see Platow et al. ${ }^{65}$ ). As well, models' characteristics such as competence, status and power have been identified as potent moderators of observational learning effects. ${ }^{4}$ In some situations, status and competence might be more potent than similarity. Field et al. ${ }^{22}$ found that verbal information about stimuli may change fears in children, but only when the information was provided by adults. Many unresolved questions remain regarding the moderating effects of relationship variables in the context of pain, including the need to investigate the impact of the social distance between model and observer. Preliminary evidence from research in mice demonstrated stronger social fear learning in socially related mice (e.g., siblings or mating partners) compared to non-related mice. ${ }^{43}$

Finally, broad sociocultural factors influence observational learning effects, as there is evidence that the cultural context affects individuals' health-related beliefs, coping with stressful life events, ${ }^{6,37}$ and pain behaviors. ${ }^{70,81}$ An important factor facilitating observational learning in humans appears to be shared perspectives or worldly schemata. A relevant distinction is that between 
independent and interdependent self-schemata, ${ }^{36,52}$ which impact upon how individuals cope with stressors. ${ }^{48}$ In independent cultures (e.g., most European countries, Australia, Canada, Great Britain, United States), individuals strive to be unique and to promote personal goals. Individuals in interdependent cultures (e.g., cultures in Africa, South America, Asia and some Middle Eastern countries such as Iran and Turkey) emphasize connectedness and the social context. ${ }^{52}$ It is reasonable to expect that observational learning effects will be much more pronounced in individuals from interdependent cultures as compared to independent cultures.

\section{Modulation by characteristics of the observer}

In the context of pain, almost no research is available examining modulation of observational learning by observer characteristics. Nevertheless, important questions can be raised concerning susceptibility of particular populations to observational learning in the context of pain. Observation of others' fearful reactions to pain-related stimuli would appear more likely to give rise to pain-related fear when observers engaged in high levels of catastrophic thinking about pain. A relevant study by Bandura and Rosenthal ${ }^{5}$ investigated the impact of prior stress or arousal in observers upon observational learning, demonstrating that moderately aroused observers displayed the most rapid and enduring fear responses to a cue signaling pain. Those who were either minimally or markedly aroused showed the weakest observational learning effects. Habitual patterns of empathic responding also might influence observational learning effects. A recent experimental study investigated the role of dispositional empathy on the effects of observational learning of placebo analgesia. Colloca and Benedetti ${ }^{12}$ found that observers reporting a high tendency to react with empathic concern to others in need after they had observed a model undergoing a beneficial treatment subsequently demonstrated the largest responses to a placebo intervention.

Documentation of the impact of observational learning has focused upon the relatively substantial changes instigated by observational learning, with relatively minimal attention to cognitive schemata serving as important moderator. Schemata related to pain are a product of one's family history of pain ${ }^{63}$ which serve as a vector for intergenerational transmission of ethnocultural belief 
systems, as these relate to personal pain-related experiences. New information is processed in the context of how it fits the existing schema. From a learning theory perspective, it is known that existing knowledge is not easily modified. Instead, exceptions to an existing rule are learnt. ${ }^{9}$ This is consistent with Piaget's theory ${ }^{64}$ regarding assimilation and accommodation. Applied in the context of pain, it is reasonable to assume that available knowledge is less likely to be changed (i.e., accommodated) by a transitory, immediate experience. New knowledge (e.g., observing one’s child encountering minor everyday pain) will be 'assimilated' into existing knowledge structures (e.g., my child often falls without serious consequences). In the case of limited prior knowledge, new information that is particularly salient may modify substantially existing knowledge structures (i.e., accommodation), and would be expected to have a greater impact on the observer's behavior (e.g., discovery of major new risk factors for a child). The social referencing literature is informative in demonstrating that infants are particularly prone to using another's responses to guide their own behavior when they encounter ambiguity or experience feelings of uncertainty toward a given object. ${ }^{32}$ For children, the dialectical interplay of assimilation and accommodation may generate increasingly more complex knowledge on pain and how to cope with pain.

Furthermore, schemata may have a crucial role in observational learning as they direct our attention to schema-relevant information, ${ }^{77}$ essentially a process of confirming pre-existing beliefs. Substantial evidence also is available demonstrating the role of selective attention in determining what is learnt and what not. ${ }^{69}$ However, almost no research is available on the role of selective attention in learning from others. In the context of pain, selective attention for behaviors of individuals in pain might strongly direct learning. Pain commands attention, whether in self-experience or when in settings where others encounter injuries or there is acute exacerbation of pain. ${ }^{30}$ Given the importance of others' faces in learning about the world ${ }^{23}$ and pain in particular, ${ }^{16,78}$ pain research should investigate the variables impacting upon selective attention to (pain) faces, and the effects of selective attention upon learning. Observational learning effects would be expected to be stronger when processing familiar others rather than strangers. Mothers allocate increased attention to their own children’s faces compared to other child and adult faces. ${ }^{31}$ Furthermore, observational learning effects 
may be stronger in individuals with high fear of pain because of preferential processing of threatening information. Also, risk groups for not profiting from observational learning should be identified. Given that faces do not capture selective attention in children with autism spectrum disorder, ${ }^{46}$ those children would be expected to show less evidence for observational learning in the context of pain. Furthermore, relationship schemata (e.g., attachment), ${ }^{54}$ which have been found to modulate attention, ${ }^{20}$ may direct future learning about pain from others.

Finally, developmental (e.g., intellectual capabilities, infant, child, adolescent stage ${ }^{34,35}$ ) and sociodemographic characteristics, such as gender, are important. The substantial gender differences associated with pain ${ }^{74}$ would be expected to have at least some basis in observational learning. ${ }^{56}$ Women report more pain models who also are more frequently female and would encounter a more supportive environment in which to express pain than would male peers. ${ }^{47}$ Gerull and Rapee ${ }^{24}$ demonstrated that gender influenced observational learning effects: girls exposed to rubber snakes and spiders showed more avoidance behavior following the observation of negative reactions of their mothers than did boys. In the context of pain, few studies are available. Goodman and McGrath ${ }^{25}$ did not find differences between boys and girls in displayed pain behaviors to a cold pressor task after observing their mother performing the task. In future studies, it would be worthwhile considering observer-model gender similarity. For example, it is reasonable to assume that observers might be more susceptible to observational learning from same-gendered models.

\section{Conclusion}

Some evidence is available on the importance of observational learning as a determinant of pain experience and behavior, but the field warrants further development. Anxiety research has been instructive as observational learning has been identified as an important pathway for developing anxiety disorders, ${ }^{57}$ and as equally important as direct and instructed fear learning pathways. ${ }^{62}$ Future studies in the context of pain should investigate the conditions under which observational learning takes place, the type of knowledge learnt during observational learning (e.g. beliefs about pain and its treatment, display rules), and when and how this knowledge is translated into overt behavior. Research 
also should examine whether particular types of pain-related beliefs or behaviors are more affected by learning from others, and whether particular environmental or observer characteristics moderate observational learning for some beliefs or behaviors and not for others.

A better understanding of these observational learning mechanisms is expected to have implications for the prevention and management of clinical pain. While speculative, observational learning influences appear to have a substantial impact on the acquisition and treatment of typical and atypical pain behavior. ${ }^{13}$ Several new avenues for clinical applications appear worth exploration. Involving the entire family in therapy (e.g., children, spouse) instead of only the pain model may enhance therapy effectiveness, by making use of observational learning effects (e.g., modelling of adequate coping behavior by family members). Some evidence is available; e.g., spouse-assisted coping skills training (including joint practice in pain coping skills) in a psychosocial intervention for chronic pain has been found to be effective in enhancing self-efficacy and coping abilities of ostheoartritis patients. ${ }^{44}$ Furthermore, as evidence is available on the "immunisation" qualities of previous exposure to non-fearful models, ${ }^{21,57}$ the application to pain-related situations needs further study. In case of existing maladaptive pain behaviors (e.g., sustained avoidance), future studies should examine whether exposure to models who have mastered fear of pain, and have re-engaged in activities is effective in reducing pain-related fear and avoidance behaviors. ${ }^{45}$ Finally, research should point out whether maximizing similarities among chronic pain group therapy members might enhance observational learning effects. 


\section{Acknowledgements}

We would like to thank Jan De Houwer for his reflective thoughts on observational learning in the context of pain and three anonymous reviewers for their valuable suggestions which have improved the manuscript. The contribution of Johan W.S. Vlaeyen was supported by an Odysseus Grant by the Fund for Scientific Research - Flanders, Belgium (FWO). The authors declare to have no conflicts of interest. 


\section{References}

1. Askew C, Field AP: Vicarious learning and the development of fears in childhood. Behav Res Ther 45:2616-2627, 2007

2. Askew C, Field AP: The vicarious learning pathway to fear 40 years on. Clin Psychol Rev 28:12491265, 2008

3. Bandura A: Influence of models reinforcement contingencies on the acquisition of imitative responses. J Pers Soc Psychol 1:589-595, 1965

4. Bandura A: Social foundations of thought and action: A social cognitive theory. Prentice Hall, Englewood Cliffs, NJ, 1986

5. Bandura A, Rosenthal TL: Vicarious classical conditioning as a function of arousal level. J Pers Soc Psychol 3:54-62, 1966

6. Berg CA, Upchurch R: A developmental-contextual model of couples coping with chronic illness across the adult life span. Psychol Bull 133:920-954, 2007

7. Blount RL, Devine KA, Cheng PS, Simons LA, Hayutin L: The impact of adult behaviors and vocalizations on infant distress during immunizations. J Ped Psychol 33:1163-1174, 2008

8. Boersma K, Linton SJ: Expectancy, fear and pain in the prediction of chronic pain and disability: A prospective analysis. Eur J Pain 10:551-557, 2006

9. Bouton, ME: A learning theory perspective on lapse, relapse, and the maintenance of behavior change. Health Psychol 19:57-63, 2000

10. Braaksma MAH, Rijlaarsdam G, van den Bergh H: Observational learning and the effects of model-observer similarity. J Educ Psychol 94:405-415, 2002

11. Bruchey AK, Jones CE, Monfils MH: Fear conditioning by-proxy: Social transmission of fear during memory retrieval. Behav Brain Res 214:80-84, 2010

12. Colloca L, Benedetti F: Placebo analgesia induced by social observational learning. Pain 144:2834, 2009

13. Craig KD: Social modeling influences on pain. In: The psychology of pain (2nd Ed.) (Sternbach, R.A., Ed.), Raven Press, New York, 1986, pp. 67-96

14. Craig KD, Lowery HJ: Heart-rate components of conditioned vicarious autonomic responses. J Pers Soc Psychol 11:381-387, 1969

15. Craig KD, Prkachin KM: Social modeling influences on sensory decision theory and psychophysiological indexes of pain. J Pers Soc Psychol 36:805-815, 1978 
16. Craig KD, Versloot J, Goubert L, Vervoort T, Crombez G: Perceiving pain in others: Automatic and controlled mechanisms. J Pain 11:101-108, 2010

17. Craig KD, Weiss SM: Vicarious influences on pain threshold determinations. J Pers Soc Psychol 19:53-59, 1971

18. De Houwer J: A conceptual and theoretical analysis of evaluative conditioning. Span J Psychol 10:230-241, 2007

19. De Houwer J: The propositional approach to associative learning as an alternative for association formation models. Learn Behav 37:1-20, 2009

20. Dewitte M, De Houwer J: Adult attachment and attention to positive and negative emotional face expressions. J Res Pers 42:498-505, 2008

21. Egliston KA, Rapee RM: Inhibition of fear acquisition in toddlers following positive modeling by their mothers. Behav Res Ther 45:1871-1882, 2007

22. Field AP, Argyris NG, Knowles KA: Who's afraid of the big bad wolf: a prospective paradigm to test Rachman’s indirect pathways in children. Behav Res Ther 39:1259-1276, 2001

23. Frischen A, Bayliss AP, Tipper SP: Gaze cueing of attention: visual attention, social cognition, and individual differences. Psychol Bull 133:694-724, 2007

24. Gerull FC, Rapee RM: Mother knows best: Effects of maternal modeling on the acquisition of fear and avoidance behavior in toddlers. Behav Res Ther 40:279-287, 2002

25. Goodman JE, McGrath PJ: Mothers' modeling influences children's pain during a cold pressor task. Pain 104:559-565, 2003

26. Goubert L, Craig KD, Buysse A: Perceiving others in pain: Experimental and clinical evidence on the role of empathy. In: The Social Neuroscience of Empathy (Ickes W and Decety J, Eds), The MIT Press, Massachusetts, 2009a, pp. 153-165

27. Goubert L, Craig KD, Vervoort T, Morley S, Sullivan MJL, Williams ACdeC, Cano A, Crombez G: Facing others in pain: the effects of empathy. Pain 118:285-288, 2005

28. Goubert L, Crombez G, De Bourdeaudhuij I: Low back pain, disability and back pain myths in a community sample: prevalence and interrelationships. Eur J Pain 8:385-394, 2004

29. Goubert L, Crombez G, Hermans D, Vanderstraeten G: Implicit attitude towards pictures of backstressing activities in pain-free subjects and patients with low back pain: an affective priming study. Eur J Pain 7:33-42, 2003 
30. Goubert L, Vervoort T, Crombez G: Pain demands attention of others: the approach/avoidance paradox [editorial]. Pain 143:5-6, 2009b

31. Grasso DJ, Moser JS, Dozier M, Simons R: ERP correlates of attention allocation in mothers processing faces of their children. Biol Psychol 81:95-102, 2009

32. Gunnar MR, Stone C: The effects of positive maternal affect on infant responses to pleasant, ambiguous, and fear-provoking toys. Child Dev 55:1231-1236, 1984

33. Guzmán YF, Tronson NC, Guedea A, Huh KH, Gao C, Radulovic J: Social modeling of conditioned fear in mice by non-fearful conspecifics. Behav Brain Res 19:173-178, 2009

34. Hatchette JE: The socialization of pain: Peer influences in adolescence. Pediatr Pain Lett 7:1-4, 2005.

35. Hatchette JE, McGrath PJ, Murray M, Finley GA: The role of peer communication in the socialization of adolescents' pain experiences: a qualitative investigation. BMC Pediatrics 8: 2, 2008

36. Heine SJ, Buchtel EE: Personality: The universal and the culturally specific. Annu Rev Psychol 60: 369-94, 2009

37. Henrich J, Henrich N: The evolution of cultural adaptations: Fijian food taboos protect against dangerous marine toxins. Proc R Soc B 2010 July 28; [epub ahead of print]

38. Hermann C: Modeling, social learning in pain. In: The encyclopedia of pain (Schmidt RF, Willis WD, Eds), Springer Publishing, Heidelberg, 2007, pp. 491-493

39. Horton RE, Riddell RRP: Mothers' facial expressions of pain and fear and infants' pain response during immunization. Infant Ment Health J 31: 397-411, 2010

40. Itier RJ, Batty M: Neural bases of eye and gaze processing: The core of social cognition. Neurosci Biobehav Rev 33:843-863, 2009

41. Jackson PL, Meltzoff AN, Decety J: How do we perceive the pain of others? A window into the neural processes involved in empathy. Neuroimage 24:771-779, 2005

42. Jensen MP, Turner JA, Romano JM: Changes after multidisciplinary pain treatment in patient pain beliefs and coping are associated with concurrent changes in patient functioning. Pain 131:38-47, 2007 43. Jeon D, Kim S, Chetana M, Jo D, Ruley HE, Lin SY, Rabah D, Kinet JP, Shin HS: Observational fear learning involves affective pain system and Ca(v)1.2 Ca2+ channels in ACC. Nat Neurosci 13:482-U105, 2010 
44. Keefe FJ, Caldwell DS, Bauwom D, Salley A, Robinson E, Timmons K, Beaupre P, Weisberg J, Helms M: Spouse-assisted coping skills training in the management of knee pain in osteoarthritis: Long-term followup results. Arthritis Care Res 12:101-111, 1999

45. Kelly VL, Barker H, Field AP, Wilson C, Reynolds S: Can Rachman's indirect pathways be used to un-learn fear? A prospective paradigm to test whether children's fears can be reduced using positive information and modeling a non-anxious response. Behav Res Ther 48:164-170, 2010

46. Kikuchi Y, Senju A, Toyo Y, Osanai H, Hasegawa T: Faces do not capture special attention in children with autism spectrum disorder: A change blindness study. Child Dev 80:1421-1433, 2009

47. Koutantji M, Pearce SA, Oakley DA: The relationship between gender and family history of pain with current pain experience and awareness of pain in others. Pain 77:25-31, 1998

48. Lam AG, Zane NWS: Ethnic differences in coping with interpersonal stressors: A test of selfconstruals as cultural mediators. J Cross Cult Psychol 35:446-459, 2004

49. Langford DJ, Tuttle AH, Briscoe C, Harvey-Lewis C, Baran IK, Gleeson P, Fischer DB, Buonara M, Sternberg WF, Mogil JS: Varying perceived social threat modulates pain behavior in male mice. J Pain 2010 Aug 5; [Epub ahead of print]

50. Leeuw M, Goossens ME, Linton SJ, Crombez G, Boersma K, Vlaeyen JWS: The fear-avoidance model of musculoskeletal pain: Current state of scientific evidence. J Behav Med 30:77-94, 2007

51. Loggia ML, Mogil JS, Bushnell MC: Empathy hurts: compassion for another increases both sensory and affective components of pain perception. Pain 136:168-176, 2008

52. Markus HR, Kitayama S: Culture, self, and reality of the social. Psychol Inq 14:277-283, 2003

53. McMurtry, C.M., Chambers, C.T., McGrath, P.J., Asp, E. When ‘don’t worry’ communicates fear: Children's perceptions of parental reassurance and distraction during a painful medical procedure. Pain 150: 52-58, 2010

54. McWilliams LA, Asmundson GJG: The relationship of adult attachment dimensions to painrelated fear, hypervigilance, and catastrophizing. Pain 127: 27-34, 2007

55. Meltzoff AN, Kuhl PK, Movellan J, Sejnowski TJ: Foundations for a new science of learning. Science 325:284-288, 2009

56. Meyers CD, Riley JL, Robinson M: Psychosocial contributions to sex-correlated differences in pain. Clin J Pain 19:225-232, 2003

57. Mineka S, Zinbarg R: A contemporary learning theory perspective on the etiology of anxiety disorders: it's not what you thought it was. Am Psychol 61:10-26, 2006 
58. Mumme DL, Fernald A, Herrera C: Infants' responses to facial and vocal emotional signals in a social referencing paradigm. Child Dev 67:3219-3237, 1996

59. Nuku P, Bekkering H: Joint attention: Inferring what others perceive (and don't perceive). Conscious Cogn 17:339-349, 2008

60. Ochsner KN, Zaki J, Hanelin J, Lodlow DH, Knierim K, Ramachandran T, Glover GH, Mackey SC: Your pain or mine: Common and distinct neural systems supporting the perception of pain in self and other. Soc Cogn Affect Neur 3:144-160, 2008

61. Olsson A, Nearing KI, Phelps EA: Learning fears by observing others: the neural systems of social fear transmission. Soc Cogn Affect Neur 2:3-11, 2007

62. Olsson A, Phelps EA: Social learning of fear. Nat Neurosci 10:1095-1102, 2007

63. Palermo TM, Chambers CT: Parent and family factors in pediatric chronic pain and disability: An integrative approach. Pain 119:1-4, 2005

64. Piaget J: Piaget's theory. In: Carmichael's manual of child psychology, Vol. 1 (Mussen PH, Ed.), John Wiley \& Sons, Inc., New York, 1970, pp. 703-732

65. Platow MJ, Grace, DM, Wilson N, Burton D, Wilson A: Psychological group memberships as outcomes of resource distributions. Eur J Soc Psychol 38:836-851, 2008

66. Poole GD, Craig K: Judgments of genuine, suppressed and faked facial expressions of pain. J Pers Soc Psychol 63:797-805; 1992

67. Preston SD, de Waal FBM: Empathy: its ultimate and proximate bases. Behav Brain Sci 25:1-72, 2002

68. Prkachin KM, Craig KD: Influencing nonverbal expressions of pain: Signal detection theory analyses. Pain 21:399-409, 1985

69. Roelfsema PR, van Ooyen A, Watanabe T: Perceptual learning rules based on reinforcers and attention. Trends Cogn Sci 14:64-71, 2010

70. Sargent C: Between death and shame: Dimensions of pain in Bariba culture. Soc Sci Med 19: 12991304, 1984

71. Schechter NL, Zempsky WT, Cohen LL, McGrath PJ, McMurtry CM, Bright NS: Pain reduction during pediatric immunizations: Evidence-based review and recommendations. Pediatrics 119:E1184E1198, 2007

72. Simon D, Craig KD, Miltner WHR, Rainville P: Brain responses to dynamic facial expressions of pain. Pain 126:309-318, 2006 
73. Singer T, Seymour B, O’Doherty J, Kaube H, Dolan RJ, Frith CD: Empathy for pain involves the affective but not sensory components of pain. Science 303:1157-1162, 2004

74. Unruh AM: Gender variations in clinical pain experience. Pain 65:123-167, 1996

75. Uono S, Sato W, Toichi M: Dynamic fearful expressions enhance gaze-triggered attention orienting in high and low anxiety individuals. Soc Behav Personal 37:1313-1326, 2009

76. Van de Riet WAC, Grezes J, de Gelder B: Specific and common brain regions involved in the perception of faces and bodies and the representation of their emotional expressions. Soc Neurosci 4:101-120, 2009

77. Vogt J, De Houwer J, Moors A, Van Damme S, Crombez G: The automatic orienting of attention to goal-relevant stimuli. Acta Psychol 134:61-69, 2010

78. Williams ACdeC: Facial expression of pain: An evolutionary account. Behav Brain Sci 25:439488, 2002

79. Wolff NJ, Darlington ASE, Hunfeld JAM, Verhulst FC, Jaddoe VWV, Moll HA, Hofman A, Passchier J, Tiemeier $\mathrm{H}$ : The association of parent behaviors, chronic pain, and psychological problems with venipuncture distress in infants: The generation R study. Health Psychol 28:605-613, 2009

80. Yamada M, Decety J: Unconscious affective processing and empathy: an investigation of subliminal priming on the detection of painful facial expressions. Pain 143:71-75, 2009

81. Zatzick DF, Dimsdale JE: Cultural variations in response to painful stimuli. Psychosom Med 52:544-557, 1990 